\title{
MicroRNA-17-5p acts as a biomarker and regulates mitochondrial dynamics in trophoblasts and endothelial cells by targeting the mitofusins Mfn1/Mfn2 in gestational diabetes mellitus
}

\author{
Type \\ Research paper
}

Keywords

Biomarker, Gestational diabetes mellitus, MicroRNA-17-5p, Mitofusin, Mitochondrial dynamic

\begin{abstract}
Introduction

Gestational diabetes mellitus (GDM) is a metabolic disease that endangers pregnant women and their offspring. Insights into biomarkers and GDM pathogenesis are crucial. Ectopic expression of microRNA-17-5p was found in GDM, but its the diagnostic value and role of miR-17-5p remain unclear.
\end{abstract}

Material and methods

Detection of miRNA microarray and quantitative PCR (qPCR) found that miR-17-5p was significantly increased and positively associated with biochemical indicators of GDM in 30 GDM plasma samples and 28 matched control plasma samples.

\begin{abstract}
Results
The area under the ROC curve was $0.827(P<0.01)$, which showed good diagnostic potential. Mitochondrial staining showed that compared with controls, trophoblasts exhibited more mitochondrial fusion and endothelial cells exhibited more mitochondrial fission in GDM than these in controls. Western blot and qPCR assays further revealed that expression of the mitofusin Mfn1/Mfn2 was lower in primary endothelial cells from GDM patients, whereas their expression was significantly higher in primary trophoblasts from GDM patients compared with those from controls. Conversely, miR-17-5p expression was higher in primary endothelial cells from GDM patients, whereas their expression was significantly lower in primary trophoblasts from GDM patients compared with those from controls. Bioinformatics and luciferase reporter assays confirmed that both Mfn1 and Mfn2 are targets of miR-17-5p. Last, decreased Mfn $1 / 2$ was observed not only to increase the apoptotic rate of primary endothelial cells from GDM, but also to reverse anti-apoptotic effects of miR-17-5p inhibitor.
\end{abstract}

Conclusions

MiR-17-5p regulates Mfn1/Mfn2-mediated mitochondrial dynamics involved in GDM. MiR-17-5p may serve as a promising biomarker and therapeutic target for GDM. 


\section{MicroRNA-17-5p acts as a biomarker and regulates mitochondrial dynamics in trophoblasts and endothelial cells by targeting the mitofusins Mfn1/Mfn2 in gestational diabetes mellitus}

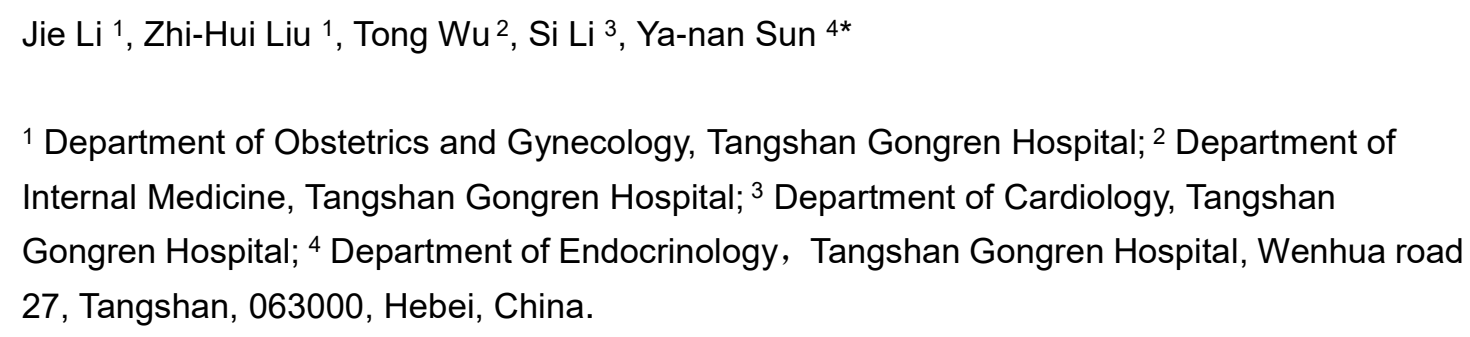

*Address for correspondence: Ya-nan Sun, MD, Department of Endocrinology, Tangshan Gongren Hospital, 27 Wenhua Road, Tangshan. E-mail: 826270793@qq.com.

\section{Abstract}

Background: Gestational diabetes mellitus (GDM) is a metabolic disease that endangers pregnant women and their offspring. Insights into biomarkers and GDM pathogenesis are crucial. Ectopic expression of microRNA-17-5p was found in GDM, but its diagnostic value and role of miR-17-5p remain unclear.

Method and result: Detection of miRNA microarray and qRT-PCR found that miR-17-5p was significantly increased and positively associated with biochemical indicators of GDM in 30 GDM plasma samples and 28 matched control plasma samples. The area under the ROC curve was $0.827(P<0.01)$, which showed good diagnostic potential. Mitochondrial staining showed that compared with controls, trophoblasts exhibited more mitochondrial fusion and endothelial cells exhibited more mitochondrial fission in GDM than these in controls. Western blot and qRT-PCR assays further revealed that expression of the mitofusin Mfn1/Mfn2 was lower in primary endothelial cells from GDM patients, whereas their expression was significantly higher in primary trophoblasts from GDM patients compared with those from controls. Conversely, miR-17-5p expression was higher in primary endothelial cells from GDM patients, whereas their expression was significantly lower in primary trophoblasts from GDM patients compared with those from controls. Bioinformatics and luciferase reporter assays confirmed that both Mfn1 and Mfn2 are targets of miR-17-5p. Last, decreased Mfn1/2 was observed not only to increase the apoptotic rate of primary endothelial cells from GDM, but also to reverse anti-apoptotic effects of miR-17-5p inhibitor.

Conclusion: MiR-17-5p regulates Mfn1/Mfn2-mediated mitochondrial dynamics involved in GDM. MiR-17-5p may serve as a promising biomarker and therapeutic target for GDM.

Key words: MicroRNA-17-5p, Biomarker, Mitofusin, Mitochondrial dynamic, Gestational diabetes mellitus

\section{Introduction}


Gestational diabetes mellitus (GDM), which is characterized by glucose intolerance with onset or first recognition during pregnancy, has become a common health problem worldwide in the context of increased obesity [1]. Its incidence is high in Asians, and its prevalence in China is $14.8 \%$ [2]. Unless properly diagnosed and treated, GDM can result in short-term and long-term adverse outcomes for both the mother and fetus, including cesarean section, shoulder dystocia, macrosomia, and neonatal hypoglycemia [3]. Although detection of GDM as early as possible is crucial to avoid poor pregnancy outcomes, the current diagnostic test for GDM is an oral glucose tolerance test (OGTT), which is performed at 24-28 weeks of gestation in compliance with the International Association of Diabetes and Pregnancy Study Groups (IADPSG). Thus, the identification of new biomarkers of GDM is anticipated to predict the early diagnosis of GDM and prevent complications, which is vital to minimize the dangers of hyperglycemia in pregnant women and their children. In addition, in order to find effective therapeutic targets, the pathophysiological mechanism of GDM requires further exploration.

Remodeling of the uterine arteries is mediated by extravillous trophoblasts, which penetrate the arterial walls and induce endothelial apoptosis, this is a key event in early pregnancy [4]. Defective remodeling is associated with pregnancy complications, such as preeclampsia and intrauterine growth restriction [5]. The diabetic environment is closely related to endothelial and vascular dysfunction [6], and recently, some miRNAs identified as potential biomarkers of GDM [7] were shown to play an important role in trophoblast-induced endothelial apoptosis [8,9]. Peng et al. reported that high glucose suppresses the viability and proliferation of HTR-8/SVneo cells through regulation of the miR-137/PRKAA1/IL-6 axis [10]. Interestingly, literatures reported that mitochondrial fission associated with hyperglycemia induces endothelial cell apoptosis and blood vessel damage $[11,12]$. Therefore, these findings urged us to explore whether these miRNAs could be the diagnostic, prognostic, and therapeutic targets in GDM.

Differential expression of some miRNAs (e.g., miR-17-5p, miR-19a-3p, miR-19b-3p, miR-20a-5p) were found in GDM, suggesting to be potential diagnostic markers [13]. However, opposing miR-17-5p expression patterns between plasma (up-regulation) and placental tissues (down-regulation) [14] from GDM patients implied that further research on the potential molecular mechanisms in GDM is still necessary.

Accordingly, in the present study, we studied the expression patterns and diagnostic value of miR-17-5p in a large cohort of GDM patients from the gynaecology and obstetrics departments of Tangshan Gongren Hospital and explored the pathophysiological role of miR-17-5p in trophoblasts and endothelial cells in GDM.

\section{Materials and Methods}

\section{Clinical specimens}

Thirty GDM-complicated pregnancies and 28 normal pregnant women as control were admitted to the gynaecology and obstetrics departments of Tangshan Gongren Hospital between May 2019 and August 2020 were included in this study. Healthy pregnant women as controls not only have normal OGTT, but also meet matched age, body mass index (BMI) and gestational age. The medical history and examination of all enrolled participants should be complete. Of course, patients with GDM who already started oral hypoglycemic drugs, and 
with history of pre-existing type 1 or $2 \mathrm{DM}$ were excluded. All neonates had a birth weight between the 10th and 90th percentile. None of the women showed signs of hypertension or any other disease. Basic clinical data of the subjects are presented in Table 1. Venous blood samples $(5 \mathrm{~mL}$ ) were centrifuged at $\sim 1,000 \times \mathrm{g}$ for $30 \mathrm{~min}$ at room temperature, and plasma was stored at $-80^{\circ} \mathrm{C}$ until subsequent analysis. GDM patients were diagnosed by OGTT at 2832 weeks of gestation as previously described [15]. All patients and controls provided written informed consent. According to the agency guidelines, all these patients had got informed consent before sample collection, and to approve the study by the Research Ethics Committee of XXXXX Hospital (the reference number is GRYY-LL-2019-21).

\section{Diagnostic criteria of GDM [15]}

The diagnosis of GDM is confirmed using the diagnostic criteria of the IADPSG as followed: fasting plasma glucose $(\mathrm{FPG}) \geq 5.1 \mathrm{mmol} / \mathrm{L}, 1 \mathrm{~h} \mathrm{PG} \geq 10.0 \mathrm{mmol} / \mathrm{L}$, or $2 \mathrm{~h} \mathrm{PG} \geq 8.5$ $\mathrm{mmol} / \mathrm{L}$ and then undergo a $75 \mathrm{~g}$ oral glucose tolerance test (OGTT).

\section{miRNA profiling in GDM plasma}

Plasma specimens were enrolled from pregnancy ( 3 women with GDM) and 3 controls matched for maternal age and pregnancy period) from the gynaecology and obstetrics departments of Tangshan Gongren Hospital. MiRNA extraction and quantification followed a protocol from miRNeasy plasma kit (Qiagen, Hilden, Germany) and an ND-1000 spectrophotometer (NanoDrop, Rockland, DE, USA). Further, microRNA-seq was performed on the Illumina HiSeq4000 SE50 (Illumina, San Diego, California, USA) after a miRNA library preparation. Differential miRNA expression analysis was performed using DESeq2 software.

\section{Isolation and culture of primary human trophoblasts and endothelial cells}

Isolation of primary first-trimester cytotrophoblasts was performed as previously described [16]. Primary human first-trimester decidual ECs were isolated using a modified method described by Grimwood et al [17]. Both primary trophoblasts and endothelial cells were cultured in RPMI 1640 culture medium ( $10 \%$ fetal bovine serum) at $37^{\circ} \mathrm{C}, 5 \% \mathrm{CO}_{2}$, and $95 \%$ humidity. Purities of primary trophoblast and endothelial cells were identified by cell immunofluorescence staining using using anti-CK7 (marker for trophoblast cells) and anti-CD31 (marker for endothelial cells) antibodies, respectively. Cell experiment has been approved by the Research Ethics Committee of XXXX Hospital (the reference number is GRYY-LL-2019-21).

\section{Transfection of anti-miR-17-5p or si-Mfn 1/2 into endothelial cells}

ECs in a 6-well plate $\left(2 \times 10^{5}\right.$ cells/well) were maintained in RPMI 1640 culture medium ( $10 \%$ fetal bovine serum, $100 \mathrm{U} / \mathrm{ml}$ penicillin and $100 \mu \mathrm{g} / \mathrm{ml}$ streptomycin). For transfection, the anti-miR-17-5p or anti-control (Shanghai Genechem Co., LTD. China) were delivered at a final concentration of $75 \mathrm{nM}$ Lipofectamine ${ }^{\circledR} 2000$ (Thermo Fisher Scientific, Inc.) following the manufacturer's instructions. Effectiveness of transfection was performed by qRT-PCR to measure the levels of miR-17-5p at $36 \mathrm{~h}$ post-transfection.

Specific small interfering RNA targeting Mfn1 (si-Mfn1) and Mfn2 (si-Mfn2) as well as corresponding control (si-con) were also purchased from RiboBio. Transfection was conducted 
using Lipofectamine 2000 (Thermo Fisher Scientific) according to the manufacturer's instructions.

\section{Real time quantitative PCR ( $q R T-P C R)$}

Total RNA from treated cells and plasma samples was extracted using RNAiso Plus reagent (Takara Bio, Inc.) and TRIzolß LS reagent (Invitrogen; Thermo Fisher Scientific, Inc.), respectively. Then, $0.5 \mu \mathrm{g}$ total RNA was reverse transcribed into cDNA using a SuperScript ${ }^{\circ}$ VILO $^{\text {TM }}$ CDNA Synthesis Kit and was then used for qRT-PCR with a SYBR Green qPCR Super Mix UDG Kit (Takara Bio, Inc.) according to the corresponding manufacturers' protocols. PCR was performed as follows: $95^{\circ} \mathrm{C}$ for 2 min followed by 40 cycles at $95^{\circ} \mathrm{C}$ for $15 \mathrm{~s}, 60^{\circ} \mathrm{C}$ for $10 \mathrm{~s}$, and $72^{\circ} \mathrm{C}$ for $10 \mathrm{~s}$. The relative expression was calculated using the $2^{-\Delta \Delta \mathrm{Ct}}$ formula and was normalized to $\mathrm{U} 6$, where $\Delta \mathrm{Ct}=\mathrm{Ct}$ related microRNA-Ct $\mathrm{U} 6$ and $\Delta \Delta \mathrm{Ct}=\Delta \mathrm{Ct}$ experimental $-\Delta \mathrm{Ct}$ control. All PCR was performed in triplicate. The primer sequences of miR-17-5p are as follows: forward 5'- GCCGCCAAAGTGCTTACA -3', reverse, 5''CAGAGCAGGGTCCGAGGTA -3'; U6: forward 5'-CTCGCTTCGGCAGCACA-3' and reverse, 5'-AACGCTTCACGAATTTGGT-3'.

\section{Mitochondrial staining}

Mitochondria were visualized with MitoTracker ${ }^{\mathrm{TM}}$ Red kit followed the instruction as previously described [18]. Images were captured by confocal microscopy (Nicon Eclipse E-800). The criteria for mitochondrial fission and fusion as followed: when fused, it can form a long rod-shaped, linear and tightly connected three-dimensional mitochondrial network; when it is split, it can form granular, dot-shaped scattered mitochondria.

\section{Cell Immunofluorescence}

Protocol of cell immunofluorescence staining was described previously [18]. Briefly, primary human trophoblasts and endothelial cells were fixed by Paraformaldehyde (4\%) and permeabilized with $0.1 \%$ Triton $\mathrm{X}-100$, then blocked with $5 \%$ BSA for $1 \mathrm{~h}$, incubated overnight with the primary antibodies anti- cytokeratin-7(CK-7) (1:100, ab68459, Abcam) and anti-CD31 $(1: 100$, ab24590, Abcam $)$ at $4^{\circ} \mathrm{C}$. Alexa Fluor 568 conjugated secondary antibodies and 4'-6-diamidino-2-phenylindole (DAPI) for staining nuclei were employed. Images were viewed by a fluorescent microscope with $400 \times$ magnification.

\section{Apoptosis assay}

Apoptotic rate of endothelial cells was measured using the Annexin-V FITC apoptosis detection kit (Sigma-Aldrich, Merck, Beijing, China). Briefly, primary endothelial cells $(2 \times$ $10^{5} /$ well) were seeded into 12-well plates and cultured for $48 \mathrm{~h}$ at $37^{\circ} \mathrm{C}$ and then resuspended in HEPES buffer with Annexin- $\mathrm{V}$ and $\mathrm{PI}$ for detection using flow cytometry (Becton Dickinson, San Jose, CA, USA).

\section{Western blot}

Western blotting assay was performed as previously described [19]. Briefly, the extracted total protein concentration was determined by a BCA assay. Total protein was separated via SDS-PAGE and then transferred onto PVDF membranes. Non-fat milk for blocking was 
followed by incubation overnight at $4^{\circ} \mathrm{C}$ with the following primary antibodies: Anti-Mfn1 (1:1,000, 66776-1-Ig, Proteintech); anti-Mfn2 (1:1,000, 67487-1-Ig, Proteintech) and anti- $\beta$-actin (1:5,000, 20536-1-AP, Proteintech) and goat anti-rabbit secondary antibodies (SA00001-1, Proteintech). The bonded proteins were visualized by chemiluminescent reaction with ECL (Amersham Pharmacia Biotech, Inc, Little Chalfont, United Kingdom).

\section{Luciferase reporter gene}

A partial sequence of the 3'-UTRs of Mfn1 and Mfn2 as well as the corresponding mutated miR-17-5p target site in the 3'-UTRs of Mfn1 and Mfn2 were each cloned into the downstream region of the firefly luciferase gene in the pGL2 Vector (Promega, Madison, WI, USA). 293T cells $\left(5 \times 10^{4}\right.$ cells/well) were seeded in 24-well plates and co-transfected with the vector (pGL2-Mfn1-WT or pGL2-Mfn1-MUT and pGL2-Mfn2-WT or pGL2-Mfn2-MUT) and miR-NC mimic or miR-17-5p mimic. After $48 \mathrm{~h}$, the cells were subjected to a dual luciferase assay (Promega, Madison, WI, USA). Detection of the binding ability of miR-17-5p and the Mfn1 or Mfn2 3'-UTRs was performed in three separate experiments. Renilla luciferase activity served as the internal control to normalized luciferase activity.

\section{Statistical analysis}

SPSS 23.0 software was used for the statistical analyses. Data are presented as means \pm standard deviations (SD), and the error bars represent SD. For continuous variables, a comparison between two groups was done using $t$-test for the normal distribution and Mann-Whitney $\mathrm{U}$ test for the non-normal distribution. Correlation test between two continuous variables were done by Pearson correlation coefficient. A receiver operating characteristic (ROC) curve was plotted for miR-17-5p to show the clinical sensitivity and specificity for possible cut-off values. Significance level was $p<0.05$.

\section{Results}

Up-regulation of miR-17-5p expression in plasma from women with GDM

Using a high-throughput human miRNA microarray, we found the miRNA expression profiles between 3 GDM plasma specimens and 3 age- and pregnancy-matched normal plasma specimens, which served as corresponding controls. The results of hierarchical clustering and qRT-PCR validation showed top 10 up-regulated miRNAs among the 6 samples (Fig. 1a and 1b).

Subsequently, we expanded the sample size (30 GDM specimens: age range from 25 to $34,29.4 \pm 3.2$ years, and responding 28 control: age range from 24 to $33,28.9 \pm 4.1$ years) to confirm increased plasma miR-17-5p expression in GDM. As presented in Fig. 1c, miR-17-5p expression was significantly higher in plasma from GDM patients than that in controls $(P=$ 0.009). Importantly, the diagnostic value of miR-17-5p in plasma from GDM patients was also verified by ROC curve analysis. The results showed that the area under the ROC curve (AUC) was 0.827 and that the sensitivity and specificity were 0.816 and 0.865 , respectively (Fig. 1d).

In addition, we also determined whether a high level of miR-17-5p was associated with biochemical parameters of GDM patients. As shown in Table 2, a significant positive correlation was observed between miR-17-5p and FBG, HbA1c, and total cholesterol in GDM 
patients. These results suggest that miR-17-5p may serve as a promising biomarker for GDM

\section{Pattern of mitochondrial dynamics in trophoblasts and endothelial cells}

Since the association between mitochondrial fission and endothelial apoptosis has been established in diabetes [20], the mechanism by which the expression of miR-17-5p whether affects trophoblast-induced endothelial cell apoptosis, as mediated by mitochondrial dynamics, was determined by visualizing mitochondrial morphology. Purities of primary trophoblast and endothelial cells were identified by cell Immunofluorescence staining using anti-CK7 and anti-CD31 antibodies, respectively (fig. 2a). The results of MitoTracker ${ }^{\mathrm{TM}}$ Red staining showed that most mitochondria in trophoblasts from GDM has a rod-like shape (fused mitochondria). In contrast, in endothelial cells from GDM, increased mitochondrial fission was observed, and the number of endothelial cells containing fragmented mitochondria was much higher in GDM patients than in controls (Fig. 2b and 2c). Furthermore, the mitochondrial fusion-related genes targeted by miR-17-5p were predicted online using TargetScan (Fig. 2d), which implied that the pattern of mitochondrial dynamics (Mitofusins, Mfn1/Mfn2) may play different roles in primary trophoblast and endothelial cells from GDM.

Mitochondrial fusion-related Mfn1 and Mfn2 expression is associated with miR-17-5p

To confirm whether Mfn1 and Mfn2 expression is associated with miR-17-5p in different cell types, we detected the expression of these proteins in primary trophoblasts and endothelial cells from GDM and control patients. Western blot and qRT-PCR analysis showed that decreased expression of Mfn1 and Mfn2 as well as increased miR-17-5p expression were observed in endothelial cells from GDM patients compared with controls (Fig. 3a-3b). Conversely, increased Mfn1 and Mfn2 expression as well as decreased miR-17-5p expression were observed in trophoblasts from GDM patients compared with controls (Fig. 3c-3d). To further determine the effect of miR-17-5p knockdown on Mfn1 and Mfn2 expression, endothelial cells were transfected with anti-miR-17-5p, and then qRT-PCR detection for confirming inhibition of its expression (Fig. 3e). Following that, the results of western blot indicated that compared with control, Mfn1 and Mfn2 expression was increased in anti-miR-17-5p-transfected endothelial cells (Fig. 3f). These data suggest that Mfn1 or/and Mfn2 could be target gene of miR-17-5p.

\section{Mfn1 and Mfn2 are targets of miR-17-5p}

To further explore the relationship between miR-17-5p and Mfn1/Mfn2, the specific binding sites of miR-17-5p in the 3'UTRs of Mfn1 and Mfn2 were predicted by the TargetScan prediction website (Fig. 4a). Moreover, the dual luciferase reporter gene assay was performed to verify the results of bioinformatic prediction (Fig. $4 b$ and $4 c$ ). Compared with the negative control (NC) mimic group, luciferase activity was significantly decreased in the miR-17-5p mimic group co-transfected with WT-Mfn1 ( $P<0.05$, Fig. 4b) or WT-Mfn2 ( $<<0.01$, Fig. 4c), while luciferase activity remained unchanged in the miR-17-5p mimic group co-transfected with the MUT-Mfn1 or MUT-Mfn2 plasmid (both $P$ > 0.05). These results demonstrated that Mfn1 and Mfn2 are target genes of miR-17-5p. 
cells

To examine whether miR-17-5p-mediated apoptotic effects on endothelial cells is Mfn1/2-dependent process, the rescue experiments were performed. We firstly showed the introduction of si- Mfn1/2 for decreased Mfn1 and Mfn2 expression in endothelial cells (Fig. 5a). Subsequently, the results of flow cytometry showed that down-regulation of Mfn1/2 expression promoted cell apoptosis in primary endothelial cells from GDM compared with si-con group; moreover, decreased miR-17-5p exerted anti-apoptotic effect on primary endothelial cells from GDM. Importantly, down-regulation of Mfn1 or Mfn2 expression dramatically reversed the inhibitory effects of anti-miR-17-5p on the apoptosis rate of endothelial cells from GDM (Fig. $5 b)$. These results indicated that Mfn1 and Mfn2 are downstream targets of miR-17-5p regulating apoptosis of endothelial cells.

\section{Discussion}

Trophoblast induction of endothelial cell apoptosis is an important mechanism in spiral artery remodeling in early normal pregnancy [21], but the mechanism in complicated pregnancies, such as in patients with GDM, has yet to be determined. MicroRNAs play a key role in many pathophysiological processes, especially by acting as circulating biomarkers and modulators of the proliferation, migration, invasion, and apoptosis of trophoblasts and endothelial cells [8]. The main objective of this study was to verify the clinical significance and application of circulating miR-17-5p in GDM patients and to elucidate the possible molecular mechanism of miR-17-5p in the regulation of mitochondrial dynamics involved in trophoblast-induced endothelial cell apoptosis. We demonstrated that circulating miR-17-5p is a promising biomarker of GDM since it targets Mfn1/Mfn2-mediated regulation of mitochondrial dynamics. This includes up-regulation of miR-17-5p expression in endothelial cells from GDM patients, which induces mitochondrial fission by decreasing Mfn1/Mfn2 expression, whereas down-regulation of miR-17-5p expression in trophoblasts from GDM patients induces mitochondrial fusion by reversing the inhibitory effects of Mfn1/Mfn2. This leads to acceleration of trophoblast-induced endothelial cell apoptosis in GDM.

It is well known that fetal trophoblasts in early pregnancy invade the luminal surfaces of the endothelium and replace the endothelial lining to create high-flow, low-resistance vessels for nutritional demands of the fetus [4]. Previous studies had reported increased miR-17-5p in plasma [13] and decreased expression in placental tissues [14] in GDM patients, which implies that ectopic expression of miR-17-5p in trophoblasts and endothelial cells is involved in aberrant remodeling of the uterine arteries in GDM. At present, the associated mechanism is still lacking, besides up-regulation of miRNA17-5p expression in plasma from GDM women, even at 16-19 weeks of pregnancy is positively correlated with insulin resistance, a risk factor of GDM. Mitochondrial dynamics mediate the regulation of cell proliferation, migration, and apoptosis associated with miRNAs [22,23]. Thus far, less is known about the role of miRNA17-5p in mitochondrial fission and apoptosis between trophoblasts and endothelial cells in GDM patients. In this study, we examined the effect of miR-17-5p ectopic expression on the regulation of mitochondrial dynamics as they relate to invading trophoblasts, which induce endothelial cell apoptosis in GDM. Our results showed that alteration of miR-17-5p expression in different cells facilitates the migration of trophoblasts and apoptosis of endothelial cells in GDM as a result of an imbalance in mitochondrial dynamics. Decreased miR-17-5p promotes 
more mitochondrial fusion in trophoblasts, while increased miR-17-5p promotes more mitochondrial fission in endothelial cells, which may be a possible mechanism of nutrient oversupply. This in turn leads to macrosomia and other fetal and maternal complications.

Offspring born to diabetic mothers are at risk of metabolic diseases associated with mitochondrial dysfunction. Mfn1/Mfn2 are potent modulators of mitochondrial metabolism and insulin signaling and play a key role in mitochondrial dynamics $[24,25]$. Some literatures indicate that Mfn1 is a major mediator contributing to glucose metabolic reprogramming in cancers [26,27]. Meanwhile, Mfn2 over-expression also leads to cell invasion in lung adenocarcinoma and gastric cancer [28, 29], implying that up-regulation of Mfn1/2 prompting more aggressive in throphoblasts, in turn accelerating the apoptosis of endothelial cells in GDM patients. Notably, a recent study showed that miR-20b suppresses mitochondrial dysfunction-mediated apoptosis to alleviate hyperoxia-induced acute lung injury by directly targeting Mfn1 and Mfn2 [30]. This is in line with our miRNA microarray, which showed up-regulation of miR-20 in plasma from GDM patients. This suggests that up-regulation of both miR-20 and miR-17-5p may play a role in endothelial cell apoptosis induced by excessive oxygen and hyperglycemia. These results presented that miR-17-5p, as a promising biomarker, was selectively expressed in GDM and targets Mfn1/Mfn2-mediated mitochondrial dynamics to intervene different cell type-dependent roles. In addition, recent report showed that miRNAs could manipulate nutrition metabolism, especially glucose and lipid metabolism, by regulating insulin signaling pathways, mitochondrial abnormalities-associated energy metabolism [31], considering the linking between miRNA17-5p-mediated mitochondrial fission and insulin resistance in GDM, prone to hypothesis that miRNA17-5p-mediated nutrition and ATP metabolisms could play a critical role in the pathophysiological mechanism, still deserving further discussion.

Overall, our findings provide convincing evidences that miR-17-5p exerts bidirectional regulatory effects on the migration of trophoblasts and the apoptosis of endothelial cells by targeting the expression of the mitofusins Mfn1/Mfn2 in GDM.

\section{Reference}

1. American Diabetes Association. Classification and Diagnosis of Diabetes: Standards of Medical Care in Diabetes-2019. Diabetes Care 2019;42:S13-S28.

2. Yuen L, Saeedi P, Riaz M, et al. Projections of the prevalence of hyperglycaemia in pregnancy in 2019 and beyond: results from the International diabetes Federation diabetes atlas, 9th edition. Diabetes Res Clin Pract 2019;157:107841-107861.

3. Filardi T, Tavaglione F, Di Stasio M, et al. Impact of risk factors for gestational diabetes (GDM) on pregnancy outcomes in women with GDM. J Endocrinol Invest 2018;41:671-676.

4. Jingjing Xu, Haiyi Liu, Yuanyuan Wu, et al. Proapoptotic effect of metalloproteinase 9 secreted by trophoblasts on endothelial cells. J Obstet Gynaecol Res 2011;37(3):187-194.

5. Brosens JJ, Pijnenborg R, Brosens IA. The myometrial junctional zone spiral arteries in normal and abnormal pregnancies: a review of the literature. Am J Obstet Gynecol 2002;187:1416-1423.

6. Dziedzic EA, Gąsior JS, Pawłowski M, et al. Vitamin D level is associated with severity of 
coronary artery atherosclerosis and incidence of acute coronary syndromes in non-diabetic cardiac patients. Arch Med Sci 2019;15(2): 359-368.

7. Abdeltawab A, Zaki ME, Abdeldayem Y, et al. Circulating microRNA-223 and angiopoietin-like protein 8 as biomarkers of gestational diabetes mellitus. Br J Biomed Sci 2020;61:1-6.

8. Liu RH, Meng Q, Shi YP, et al. Regulatory role of microRNA-320a in the proliferation, migration, invasion, and apoptosis of trophoblasts and endothelial cells by targeting estrogen-related receptory. J Cell Physiol 2018;234(1):682-691.

9. Ji YT, Zhang WF, Yang J, et al. MiR-193b inhibits autophagy and apoptosis by targeting IGFBP5 in high glucose-induced trophoblasts. Placenta 2020;101:185-193.

10. Peng HY, Li MQ, Li HP. High glucose suppresses the viability and proliferation of HTR $8 / \mathrm{SVneo}$ cells through regulation of the miR-137/PRKAA1/IL-6 axis. Int J Mol Med 2018;42:799-810.

11. Hao Y, Liu HM, Wei $X$, et al. Diallyl trisulfide attenuates hyperglycemia-induced endothelial apoptosis by inhibition of Drp1-mediated mitochondrial fission. Acta Diabetol 2019;56(11):1177-1189.

12. Ma LJ, Zhang ZW, Dong K, et al. TWIST1 alleviates hypoxia-induced damage of trophoblast cells by inhibiting mitochondrial apoptosis pathway. Exp Cell Res 2019;385(2):111687-111696.

13. Cao YL, Jia YJ, Xing BH, et al. Plasma microRNA-16-5p,-17-5p and -20a-5p: novel diagnostic biomarkers for gestational diabetes mellitus. J Obstet Gynaecol Res 2017;43: 974-981.

14. Tang L, Li P, Li L. Whole transcriptome expression profiles in placenta samples from women with gestational diabetes mellitus. J Diabetes Investig 2020;11(5):1307-1317.

15. Sun DG, Tian S, Zhang L, et al. The miRNA-29b is downregulated in placenta during gestational diabetes mellitus and may alter placenta development by regulating trophoblast migration and invasion through a HIF3A-dependent mechanism. Front Endocrinol 2020;11:169-178.

16. Cartwright JE, Kenny LC, Dash PR, et al. Trophoblast invasion of spiral arteries: a novel in vitro model. Placenta 2002;23:232-235.

17. Grimwood J, Bicknell R, Rees MC. The isolation, characterization and culture of human decidual endothelium. Hum Reprod 1995;10(8):2142-2158.

18. Ma D, Zheng B, Liu HL, et al. Klf5 down-regulation induces vascular senescence through elF5a depletion and mitochondrial fission. PLoS Biol 2020;18(8):e3000808.

19. Ma D, Zheng B, Suzuki T, et al. Inhibition of KLF5-Myo9b-RhoA Pathway-Mediated Podosome Formation in Macrophages Ameliorates Abdominal Aortic Aneurysm. Circ Res 2017;120(5):799-815.

20. Bhatt MP, Lim YC, Kim YM, et al. C-peptide activates AMPKa and prevents ROS-mediated mitochondrial fission and endothelial apoptosis in diabetes. Diabetes 2013;62(11):3851-3862.

21. Sandra VA, Guy SJW, Philip RD, et al. Uterine spiral artery remodeling involves endothelial apoptosis induced by extravillous trophoblasts through Fas/FasL interactions. Arterioscler Thromb Vasc Biol 2005;25(1):102-118.

22. Yan Y, Ma Z, Zhu J, et al. miR-214 represses mitofusin-2 to promote renal tubular 
apoptosis in ischemic acute kidney injury. Am J Physiol Renal Physiol 2020;318(4):878-887.

23. Channakkar AS, Singh T, Pattnaik B, et al. MiRNA-137-mediated modulation of mitochondrial dynamics regulates human neural stem cell fate. Stem Cells 2020;385(5):683-697.

24. Gonzalez-Ibanez AM, Ruiz LM, Jensen E, et al. Erythroid Differentiation and Heme Biosynthesis Are Dependent on a Shift in the Balance of Mitochondrial Fusion and Fission Dynamics. Front Cell Dev Biol 2020;8:592035-592049.

25. Bruna MG, Thiago SM, Karen FC, et al. Mice born to females with oocyte-specific deletion of mitofusin 2 have increased weight gain and impaired glucose homeostasis. Mol Hum Reprod 2020 26(12):938-952.

26. Liu X, Feng C, Wei G, et al. Mitofusin1 Is a Major Mediator in Glucose-Induced Epithelial-to-Mesenchymal Transition in Lung Adenocarcinoma Cells. Onco Targets Ther 2020 13: 3511-3523

27. Zhang Z, Li TE, Chen M, et al. MFN1-dependent alteration of mitochondrial dynamics drives hepatocellular carcinoma metastasis by glucose metabolic reprogramming. $\mathrm{Br} J$ Cancer 2020 122(2): 209-220.

28. Lou Y, Li R, Liu J, et al. Mitofusin-2 over-expresses and leads to dysregulation of cell cycle and cell invasion in lung adenocarcinoma. Med Oncol 2015 32(4): 132-141.

29. Fang CL, Sun DP, Chen HK, et al. Overexpression of Mitochondrial GTPase MFN2 Represents a Negative Prognostic Marker in Human Gastric Cancer and Its Inhibition Exerts Anti-Cancer Effects. J Cancer 2017 8(7): 1153-1161.

30. Mu G, Deng Y, Lu Z, et al. miR-20b suppresses mitochondrial dysfunction-mediated apoptosis to alleviate hyperoxia-induced acute lung injury by directly targeting Mfn1 and Mfn2. Acta Biochim Biophys Sin (Shanghai) 2020;gmaa161.

31. Du H, Zhao Y, Li H, et al. Roles of MicroRNAs in Glucose and Lipid Metabolism in the Heart. Front Cardiovasc Med 2021;8 : 716213-716224.

\section{Acknowledgments}

We thank Prof. Dong Ma for technical assistance.

\section{Author contributions}

L. J., L. Z. H., S. Y. N., W. T., L. S., Z. L. C. and O. Y. X. L. performed the experiments and analyzed the data;

L. J., S. Y. N. and L. Z. H. conceived and supervised the study; L. J. and S. Y. N. wrote the manuscript.

\section{Funding}

This work was supported by Medical Science Research Projects of Hebei Provincial Health Commission (No. 20201495).

\section{Availability of data and materials}

All data produced or analyzed during this study are included in this published article. 


\section{Declarations}

\section{Ethics approval and consent to participate}

Venous blood samples, isolation and culture of primary human trophoblasts and endothelial cells were obtained from patients undergoing surgical resection upon informed consent and approval by the Research Ethics Committee of Tangshan Gongren Hospital (approval number: GRYY-LL-2019-21).

\section{Consent for publication \\ Not applicable.}

\section{Competing interests}

The authors state that they have no competing interests.

\section{Figure legends}

Figure 1 Up-regulation of miR-17-5p expression in the plasma from GDM patients. a, Hierarchical clustering shows distinguishable miRNA expression (top 10 up-regulated miRNAs) among the 6 samples ( 3 GDM plasma specimens and 3 age- and pregnancy-matched normal plasma specimens, which served as corresponding controls). b, qRT-PCR for verification of different miRNAs expressed in the six samples. ${ }^{*} P<0.05$ and ${ }^{* *} P<0.01$ vs. control, $n=3$ for each group. c, qRT-PCR to detect the miR-17-5p level in plasma from GDM patients $(n=30)$ and controls ( $n=28$ ). ${ }^{* *} P<0.01$ vs. control. d, Receiver operating characteristic (ROC) curve of circulating miR-17-5p was constructed to predict the diagnostic potential of miR-17-5p for GDM.

Figure 2 Mitochondrial dynamics of trophoblasts and endothelial cells. a, Trophoblasts were derived from primary human first-trimester extravillous trophoblasts, while primary HUVECs were isolated from fresh umbilical cord veins after digestion with collagenase. Representative images of primary trophoblast and endothelial cells purities identified by cell immunofluorescence staining using anti-CK7 (marker for trophoblast cells) and anti-CD31 (marker for endothelial cells) antibodies, respectively. Scale bars $=50 \mu \mathrm{m}$. b. MitoTracker ${ }^{\mathrm{TM}}$ Red-stained mitochondria with DAPI for nuclear staining (blue) were observed in primary trophoblasts and endothelial cells from GDM and Control women. Scale bars $=10 \mu \mathrm{m}$. c, Statistical analysis for the percentage of cells containing fused and fragmented mitochondria from more than 100 cells between two groups. ${ }^{* *} \mathrm{P}<0.01$ and ${ }^{* * *} \mathrm{P}<0.001$ vs. control, $\mathrm{n}=3$ for each group. $\mathbf{d}$, The miR-17-5p-target regulatory network was constructed based on the TargetScan website (http://www.targetscan.org/vert_72/).

Figure 3 Association between abnormal expression of Mfn1, Mfn2, and miR-17-5p in trophoblasts and endothelial cells. a, Western blot for the detection of Mfn1 and Mfn2 expression in primary endothelial cells from GDM and control patients. $\beta$-actin served as a loading control. b, qRT-PCR to detect the miR-17-5p level in primary endothelial cells from GDM and control patients. ${ }^{* *} \mathrm{P}<0.01$ vs. control. c, Western blot for the detection of Mfn1 and Mfn2 expression in primary trophoblasts from GDM and control patients. d, qRT-PCR to detect 
the miR-17-5p level in primary trophoblasts from GDM and control patients. ${ }^{*} P<0.05$ vs. control. e, RT-qPCR for the decreased miR-17-5p expression in anti-miR-17-5p-transfected endothelial cells compared to anti-control-transfected endothelial cells. ${ }^{* * *} \mathrm{P}<0.001 \mathrm{vs}$. control. f, Western blotting assay for expression of Mfn1, Mfn2 in endothelial cells treated as above. $\beta$-actin served as a loading control.

Figure 4 MiR-17-5p targets Mfn1 and Mfn2 expression. a, The 3'-UTRs (untranslated regions) sequence for the binding of miR-17-5p to Mfn1/Mfn2. $\mathbf{b}$ and $\mathbf{c}, 293 \mathrm{~A}$ cells were transfected with the reporter directed by the Mfn1 and Mfn2 3'UTR containing the miR-17-5p binding site, and luciferase activity was measured. ${ }^{*} P<0.05$ and ${ }^{* *} \mathrm{P}<0.01 \mathrm{vs}$. NC mimic group.

Figure $5 \mathrm{Mfn} 1 / 2$ expression silencing reverses the anti-apoptosis of miR-17-5p inhibitor in primary endothelial cells from GDM. a, The protein level of Mfn $1 / 2$ was detected in anti-miR-17-5p-transfected endothelial cells from GDM with Mfn1 and Mfn2 silencing or without by Western blotting assay. $\mathbf{b}$, The apoptosis rates were also analysed in above condition by flow cytometry with PI/AnnexinV-FITC. Transfection of anti-control (blue) and antimiR-17-5p (red) in cells indicated different color column. Data were shown as mean $\pm S D$, ${ }^{*} P<$ 0.05 and ${ }^{* *} P<0.01$ vs. control or anti-miR-17-5p group. 
Table 1 Basic clinical data of the subjects in GDM and Control group

\begin{tabular}{|c|c|c|c|}
\hline Parameter & GDM $(\mathrm{n}=30)$ & Control $(\mathrm{n}=28)$ & P value \\
\hline Age (years) & $29.4 \pm 3.2$ & $28.9 \pm 4.1$ & 0.814 \\
\hline BMI (kg/m $\left.{ }^{2}\right)$ & $23.8 \pm 0.91$ & $23.6 \pm 1.08$ & 0.862 \\
\hline FBG $(\mathrm{mmol} / \mathrm{L})$ & $8.11 \pm 1.23$ & $4.85 \pm 0.77$ & $<0.001$ \\
\hline 1h-PPBG (mmol/L) & $12.3 \pm 1.34$ & $8.94 \pm 0.66$ & $<0.001$ \\
\hline $\mathrm{HbA1c} \mathrm{( \% )}$ & $8.97 \pm 1.4$ & $4.17 \pm 1.1$ & $<0.001$ \\
\hline Total cholesterol (mmol/L) & $5.92 \pm 1.34$ & $4.33 \pm 1.01$ & $<0.01$ \\
\hline $\mathrm{HDL}(\mathrm{mmol} / \mathrm{L})$ & $1.22 \pm 0.25$ & $1.16 \pm 0.21$ & 0.103 \\
\hline $\mathrm{LDL}(\mathrm{mmol} / \mathrm{L})$ & $2.59 \pm 0.53$ & $2.77 \pm 0.46$ & 0.029 \\
\hline TGs $(\mathrm{mmol} / \mathrm{L})$ & $2.31 \pm 0.68$ & $1.92 \pm 0.51$ & 0.087 \\
\hline
\end{tabular}

Note: Data was shown as mean \pm standard deviation. BMI, body mass index; FBG, fasting blood glucose; PPBG, post-prandial blood glucose; HbA1c, glycated hemoglobin; LDL, low density lipoprotein cholesterol; HDL, high density lipoprotein cholesterol; TGs, triglycerides.

Table 2 Association between miR-17-5p levels and the biochemical parameters in GDM patients

\begin{tabular}{|c|c|c|c|c|c|c|c|c|}
\hline \multicolumn{2}{|c|}{ Parameters } & FBG & 2h-PPBG & HbA1c & Total cholesterol & LDL & HDL & TGs \\
\hline \multirow{2}{*}{ miR-17-5p } & $\mathrm{r}$ & 0.473 & 0.412 & 0.558 & 0.486 & 0.31 & -0.16 & 0.23 \\
\cline { 2 - 9 } & $\mathrm{P}$ & $<0.001$ & $<0.001$ & 0.007 & $<0.001$ & 0.023 & 0.096 & 0.037 \\
\hline
\end{tabular}

Note: FBG, fasting blood glucose; PPBG, post-prandial blood glucose; HbA1c, glycated hemoglobin; LDL, low density lipoprotein cholesterol; HDL, high density lipoprotein cholesterol; TGs, triglycerides. 
A

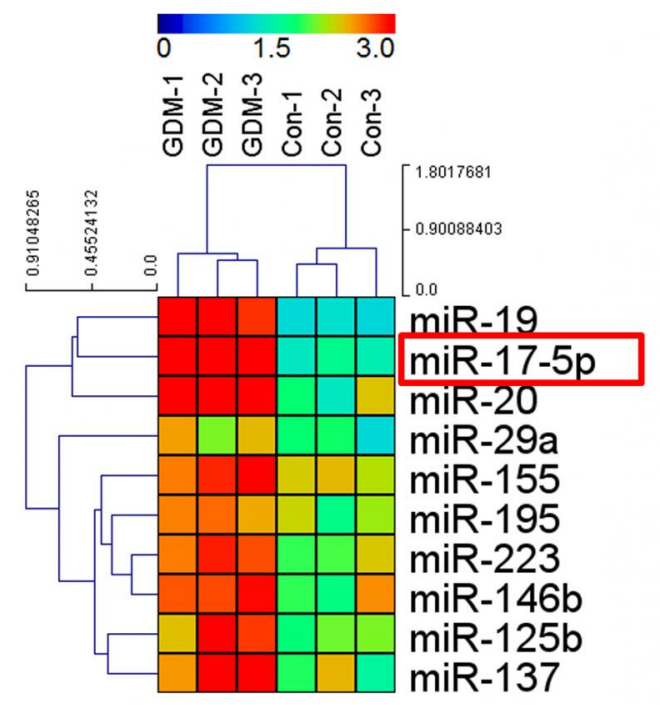

B

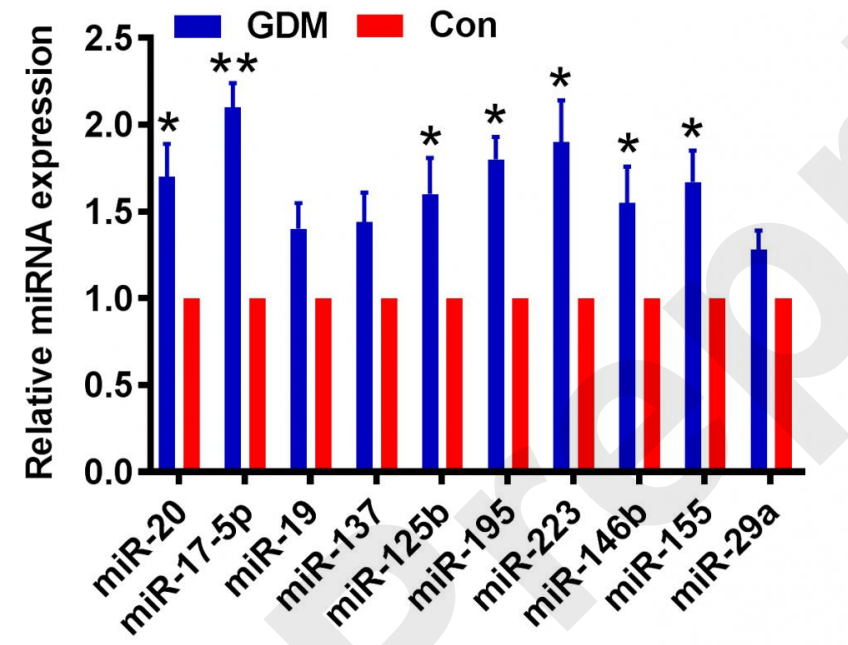

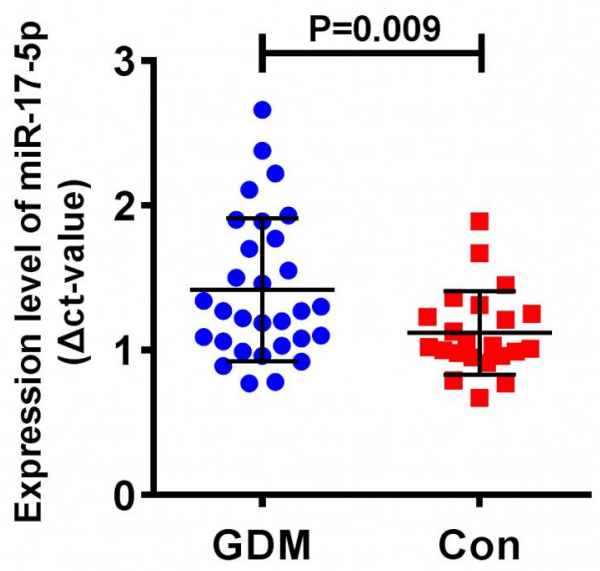

D

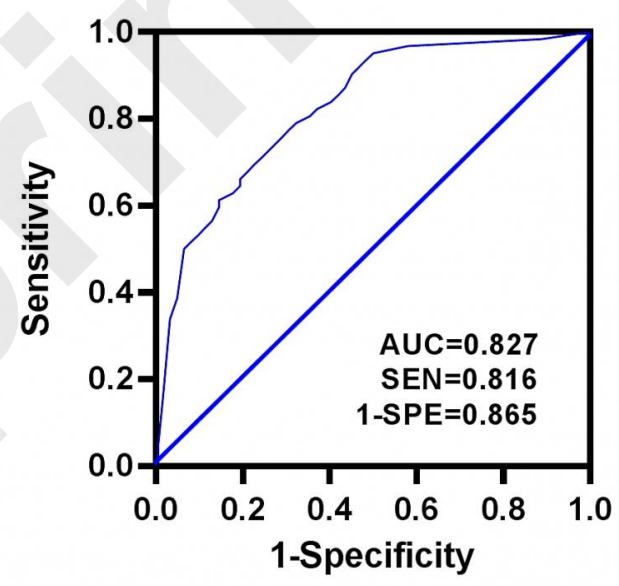


A

D

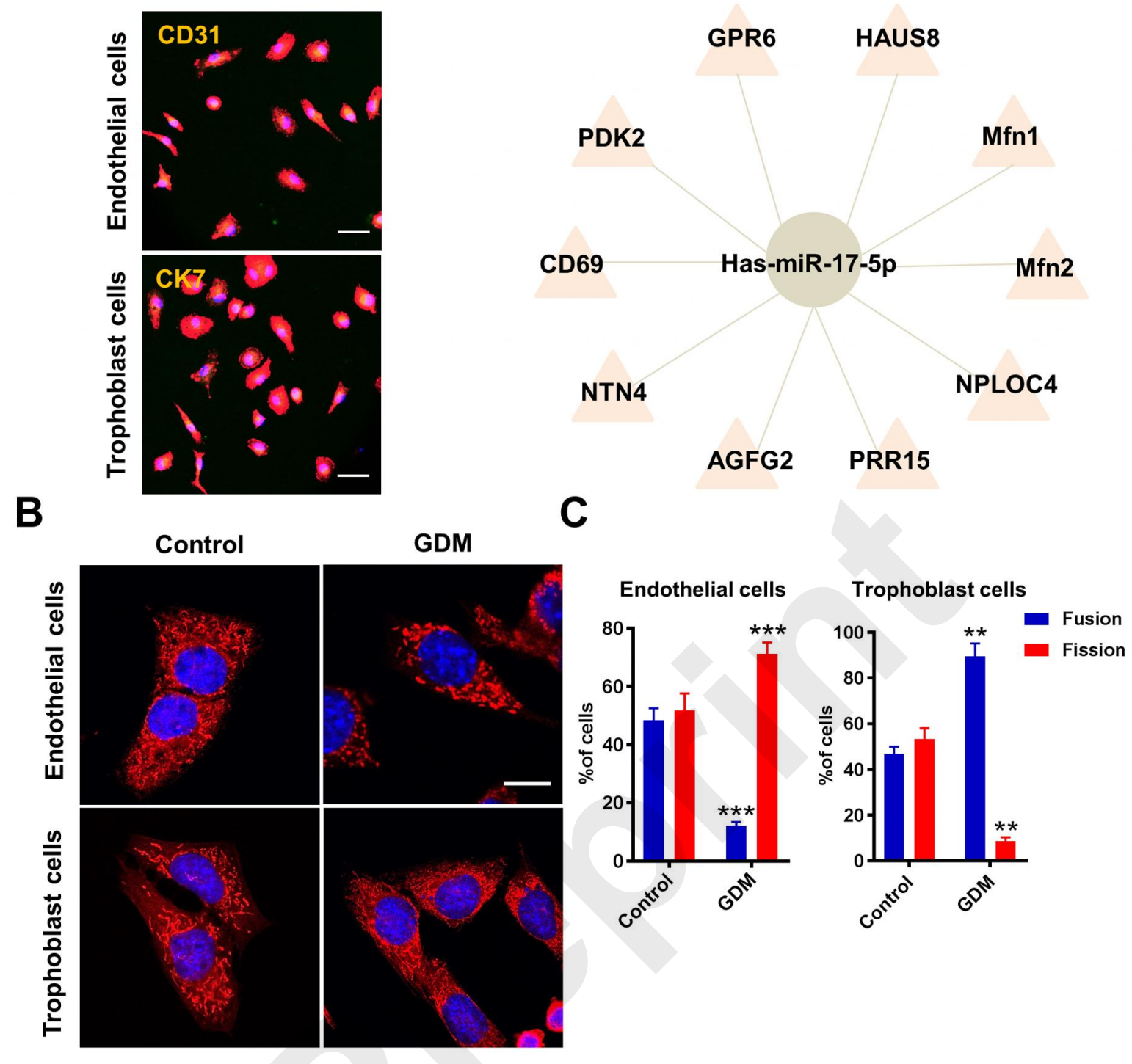


A

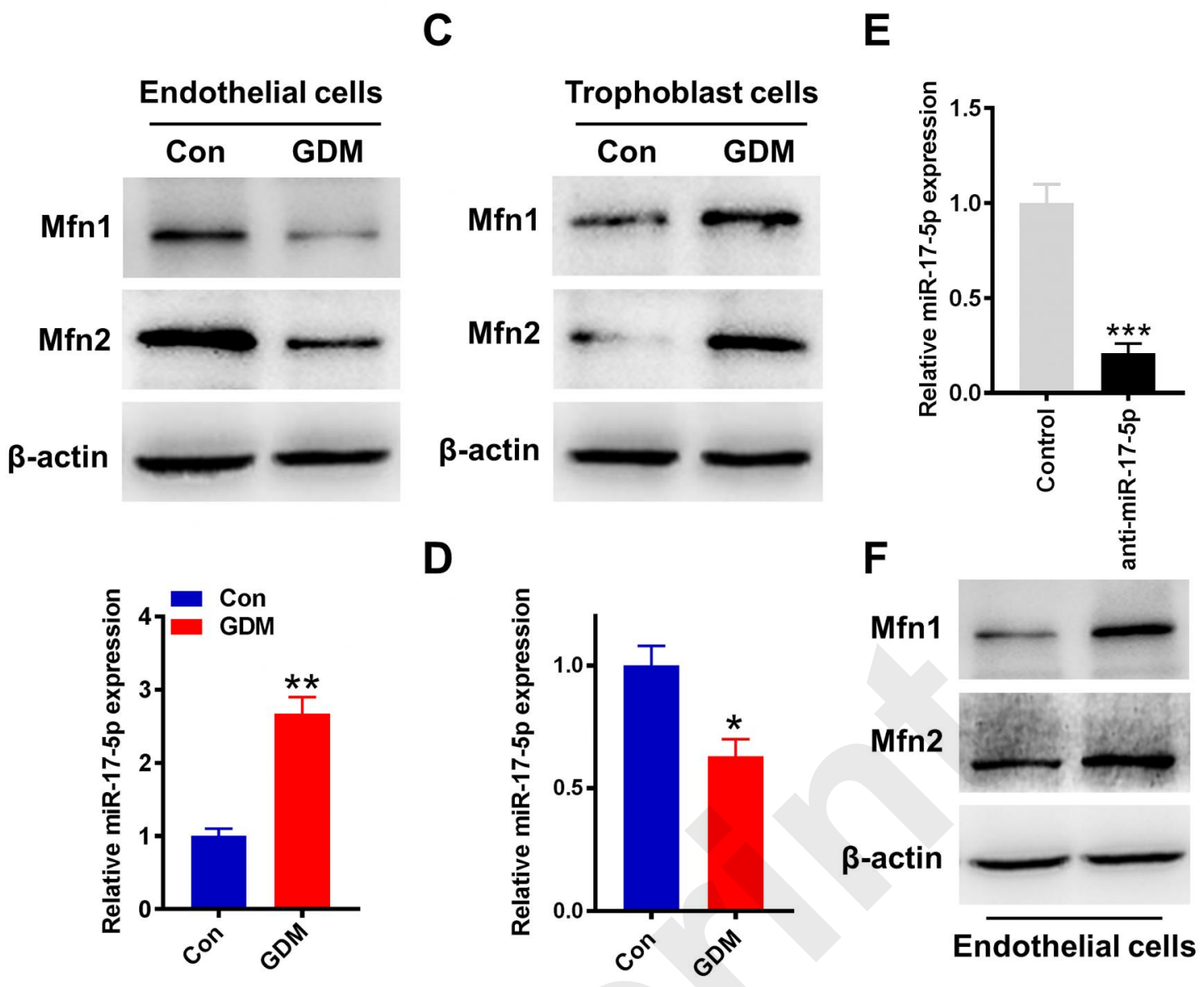


A

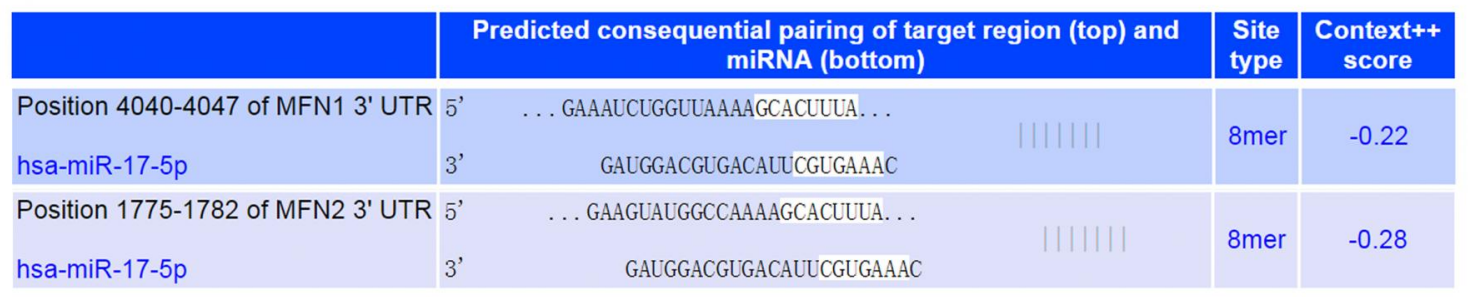

B

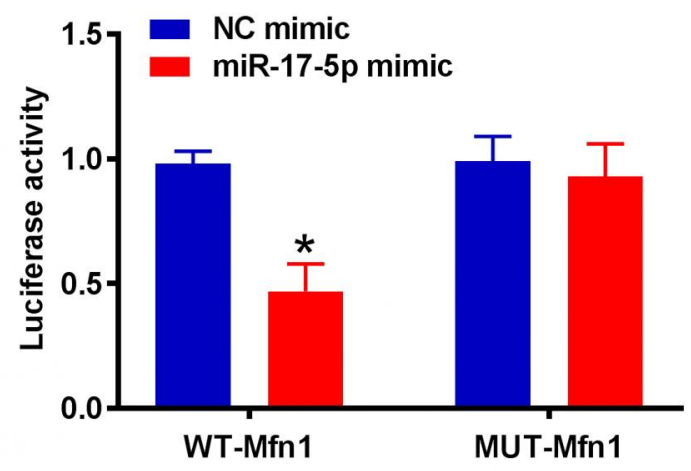

C

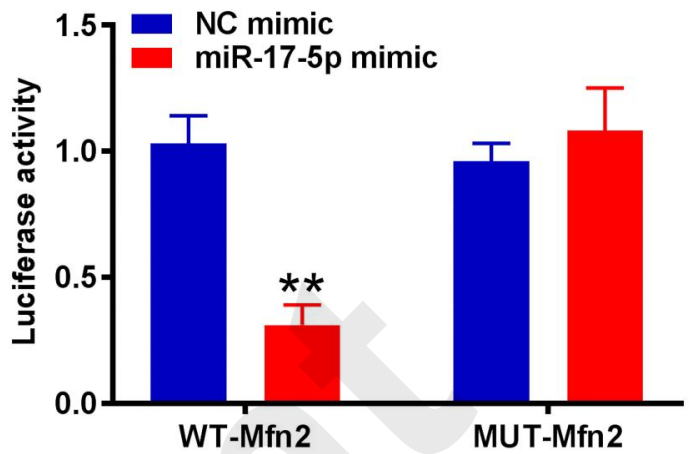


A

B
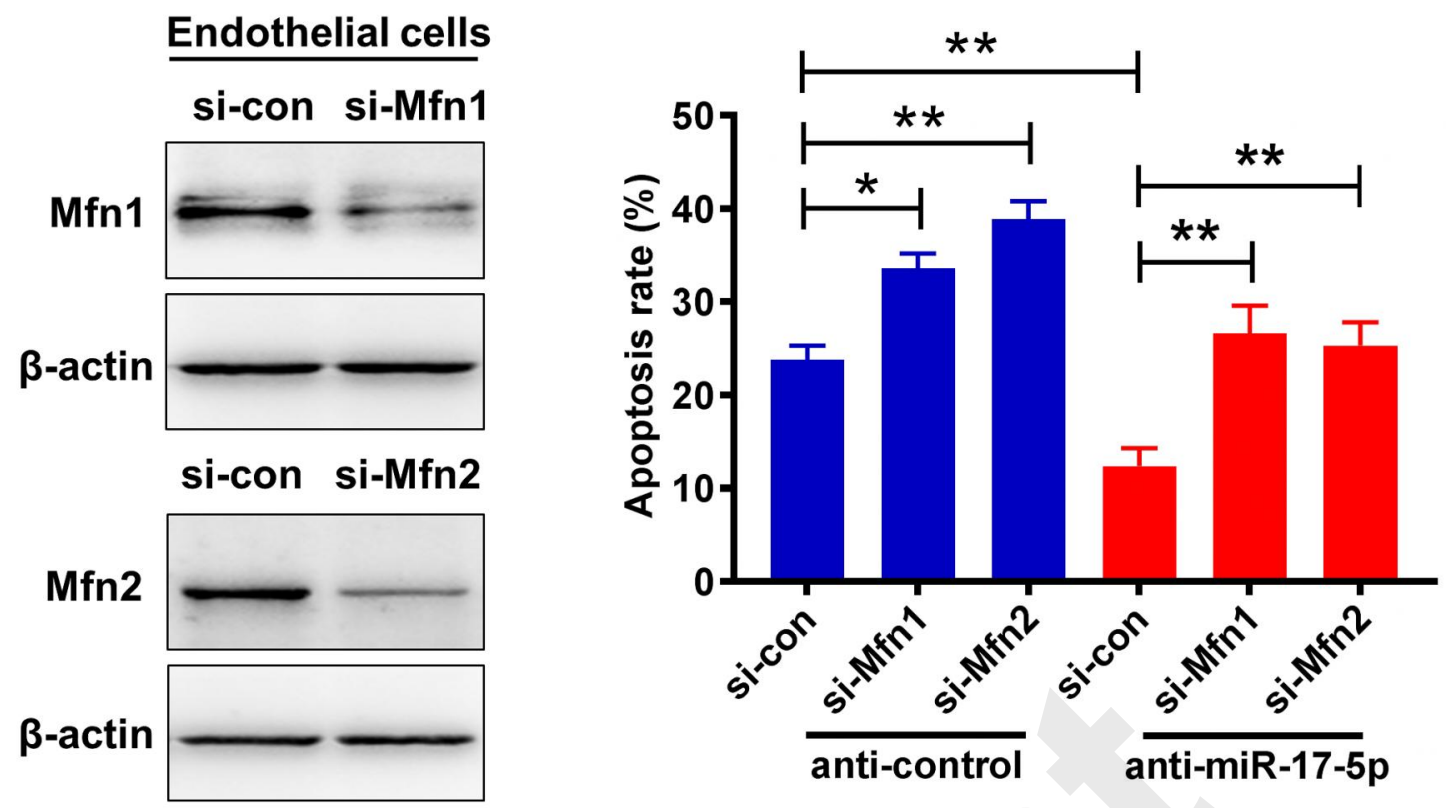